\title{
Potenciación de la inteligencia motriz para la resolución de situaciones motrices
}

\author{
William Andrés Garzón Venegas ${ }^{1}$
}

Recibido 10 de agosto de 2019 - Aceptado 01 de noviembre de 2019

\section{RESUMEN}

Este artículo presenta los resultados de la investigación que tuvo como objetivo la potenciación de los elementos constitutivos de la inteligencia motriz como determinantes en la resolución de situaciones motoras en jóvenes de media vocacional, de una institución educativa ubicada en la ciudad de Bogotá, Colombia. Frente al problema, se planteó el alejamiento de la cultura del movimiento por parte de los adolescentes, la cual incide de manera significativa en la frustración que experimentan al no poder resolver una situación motora adecuadamente en su cotidianidad. El método seleccionado fue de carácter mixto; desde lo cualitativo se implementó una investigación acción y desde lo cuantitativo un estudio preexperimental. La muestra la conformaron 28 estudiantes adolescentes de grado undécimo y 3 evaluadores. Se aplicaron 2 test validados, una encuesta diagnostica y una guía de aprendizaje para el desarrollo de la inteligencia motriz. Para el análisis de la información cuantitativa se ejecutó un diseño estadístico en Excel de los resultados obtenidos en los tests, la encuesta y la guía de aprendizaje con su respectivo análisis cualitativo. Como resultado, se pudo validar el desarrollo la inteligencia motriz con relación a la resolución de problemáticas motrices, generando niveles de desarrollo motor en porcentaje alto y superior del 79,6 \% y básico, bajo e inferior del 20,4\%, y presentando además un promedio general del 4,2 en los adolescentes impactados en la capacitación para solucionar problemas motrices en la cotidianidad, concluyendo el estudio satisfactoriamente con el alcance del objetivo general del proyecto.

Palabras clave: inteligencia motriz, elementos constitutivos, situaciones motrices, resolución.

\section{Fostering Motor Intelligence for the Resolution of Motor Situations}

\section{ABSTRACT}

This article presents the results of a research study aimed at strengthening the constituent elements of motor intelligence as determinants in the resolution of motor situations among vocational high school students at an educational institution located in the city of Bogota, Colombia. Faced with this problem, it was proposed that adolescents have moved away from the culture of the movement, and this has had a significant impact on the frustration they experience when they cannot adequately resolve a motor situation in their daily lives. The method selected was mixed, including qualitative action research and a quantitative pre-experimental study. The sample consisted of 28 eleventh grader and 3 evaluators. Two validated tests were applied, as well as a diagnostic survey and a learning

I Licenciado en Educación Física de la Universidad Pedagógica Nacional, Especialista en Pedagogía de la Lúdica de la Fundación Universitaria Los Libertadores y Maestrante en Educación de la Fundación Universitaria Los Libertadores. wagarzonv@libertadores.edu.co 
guide for the development of motor intelligence. To analyze the quantitative information, a statistical design in Excel was executed for the results obtained from the tests, the survey and the learning guide with their respective qualitative analysis. As a result, it was possible to validate the development of motor intelligence in relation to the resolution of motor problems, generating high and superior levels of motor development at $79.6 \%$ and basic, low and inferior levels at $20.4 \%$, while presenting a general average of 4.2 in the students that underwent training to solve daily motor problems. Therefore, the study concluded satisfactorily considering the scope of the project's general objective.

Key words: motor intelligence, constituent elements, motor situations, resolution.

La educación corporal es una disciplina contemporánea, que ha sido invitada a construir nuevos conocimientos disciplinares y pedagógicos con los cuales se gestarán subjetividades que tomen posición frente a la dinámica del capitalismo y la era de la globalización. A través del movimiento, el ser humano se ve infiltrado por diversas tecnologías, las cuales le están impidiendo su esencia como individuo, exponiéndolo a una mutación hacia lo cibernético y lo virtual (Sibilia, 2006). Es así como emerge el aumento de patologías por falta del movimiento, postura inadecuadas, mala alimentación, falta de inteligencia motriz y de motricidad, corporeidad dimensión lúdica y otras que son derivación del uso tecnológico.

Paralelamente, es necesario enfocar el campo de la educación corporal desde el desarrollo de la estructura de la corporeidad como un elemento constitutivo de la motricidad humana (Benjumea, 2009). Comprenderla desde esta dinámica es tener la capacidad de asimilar el cuerpo como un todo, un corpus que se relaciona en la sociedad de manera significativa; desde el impacto de actos conscientes, con los cuales logra un papel participativo en ella, a la vez que deja huella es la estructura social, configurándose como un sujeto que manifiesta sensibilidad, se desarrolla y se configura en el escenario de globalización. En resumidas cuentas, con esta habilidad corporal cabe agregar el desarrollo de los demás elementos de la motricidad: intencionalidad, temporalidad, espacialidad, acto motor, y percepción, habilidades determinantes para la solución de problemas motrices que se presenten en la cotidianidad.

Luego, el planteamiento del problema tenido en cuenta por los intereses del proyecto; para Hernández (20I4), todo comienza cuando el 
investigador se familiariza con el tema a trabajar; en este caso, la temática se centra en el fortalecimiento de los elementos constitutivos de la inteligencia motriz como determinantes en la resolución de situaciones motoras en jóvenes de media vocacional. Una vez adentrados en el tema del proyecto, el planteamiento cualitativo comprende determinar el propósito y los objetivos, la pregunta de investigación, la justificación, la viabilidad y una contextualización general, también conocida como la introducción. Todo lo anterior, relacionado y articulado con el tema central del proyecto.

Desde la perspectiva del planteamiento del problema, cabe indicar el impacto de los siguientes datos estadísticos y teorías. Un estudio realizado en la Universidad Nacional de Colombia y publicado en la Revista de Salud Pública sobre sedentarismo, el uso masivo de la tecnología y el impacto en la salud de los adolescentes. Fueron encuestados 932 adolescentes, lo que evidencia que los adolescentes en la actualidad muestran interés en el uso de la tecnología — ver televisión, estar en internet y jugar video juegos- y dejan como última prioridad realizar actividad física.

Figura $n^{\circ} I$. Proporción de adolescentes según falta de actividad

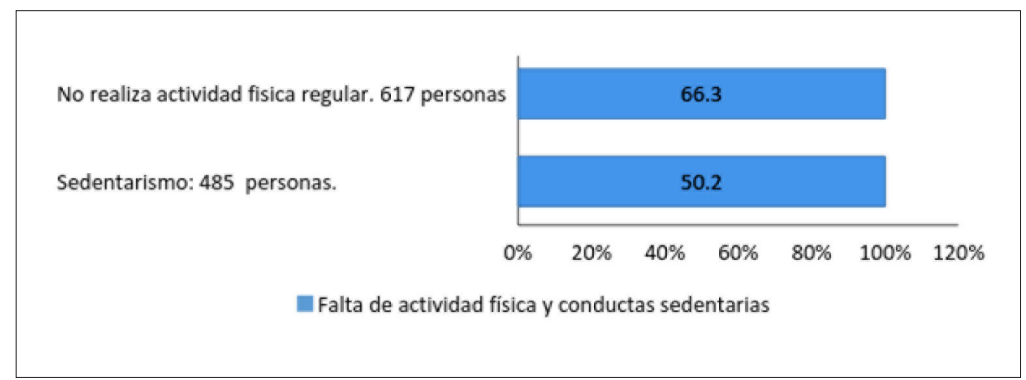

Fuente: elaboración propia (2019).

* Falta de actividad física = menos de 3 días a la semana durante 30 minutos; Sedentarismo $=$ más de 2 horas dedicado a ver TV, jugar video-juegos, Internet Fuente: Universidad Nacional de Colombia. (2014). Actividad física y sedentarismo: Determinantes sociodemográficos, familiares y su impacto en la salud del adolescente. Revista de Salud Pública.

El estudio muestra que el 66,3\% de los adolescentes de la muestra reflejó un nivel de actividad física menor al recomendado, es decir, menos de 3 días en la semana, con una duración de 30 minutos o más. 
Cuando se evaluó el tiempo que los adolescentes invierten en estar sedentarios, observando televisión o con los videos juegos, más de la mitad de la muestra de los adolescentes reportó dedicarse a este tipo de actividad más de 2 horas diarias, lo que demuestra que el interés de los adolescentes es tecnológico y no sus hábitos recomendados de actividad física. Lo cual trae consigo un factor de riesgo para su estado físico: un incremento en sobrepeso, obesidad y un estado de salud inadecuado.

En la misma línea, la Autoridad de Educación para la Salud — Health Development Agency (HDA) — aconseja la acumulación de una hora de actividad física por día de, al menos, intensidad moderada (es decir, el equivalente de caminar a paso ligero), a través de formas de vida recreativas y estructuradas. Una recomendación secundaria es que los niños y adolescentes participen en actividades que ayuden a desarrollar y mantener la salud músculo-esquelética en al menos dos ocasiones por semana (Fairclough \& Stratton, 2005). Además, cabe agregar otro beneficio desde lo cognitivo: la relación entre ejercicio y cognición es un tema importante de investigación que recientemente comenzó a desentrañarse (Valter, 2016). Comprobó él la relación entre las habilidades motoras, la función cognitiva y el rendimiento escolar en 45 estudiantes de 8 a I 4 años de edad. Lo cual evidencia el gran beneficio del ejercicio para el cerebro y sus diferentes demandas en lo social, no solo en la escuela.

Por otra parte, la encuesta bienal de culturas que realiza la Alcaldía Mayor de Bogotá, junto con la Secretaría de Cultura, Recreación y Deporte, a 19 localidades en zona residencial urbana estratificada de Bogotá, teniendo como muestra a I5.674 personas de I3 años en adelante, en la que se incorporan elementos estadísticos y de análisis frente a la cultura, el arte, el patrimonio el deporte, los hábitos de recreación, entre otros, evidencia en sus resultados con relación a la práctica del deporte para el 2015 que: 
Figura 2. Encuesta sobre la práctica de actividad física y deporte.

\section{b Actividad física y deporte 끈}

\section{P105. ¿Practica actualmente algún deporte?}

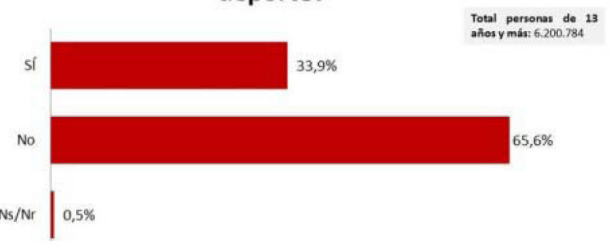

Fuente: Secretaría de Cultura, Recreación y Deporte. Encuesta Bienal de Culturas (2015).

Desde lo anterior se puede inferir que el 33,9\% de personas en Bogotá practican con frecuencia algún tipo de actividad deportiva, índice realmente bajo, ni siquiera la mitad de la población bogotana se muestra interesada por la práctica deportiva, lo que demuestra un frustración y falta de interés frente al deporte, lo que nos lleva a reiterar que existe una deserción deportiva en los adolescentes, y que ello no pronostica un buen desarrollo motor que les de la confianza necesaria para estar inmersos en escenarios deportivos.

Figura 3. Encuesta sobre la práctica deportiva según la edad de las personas.

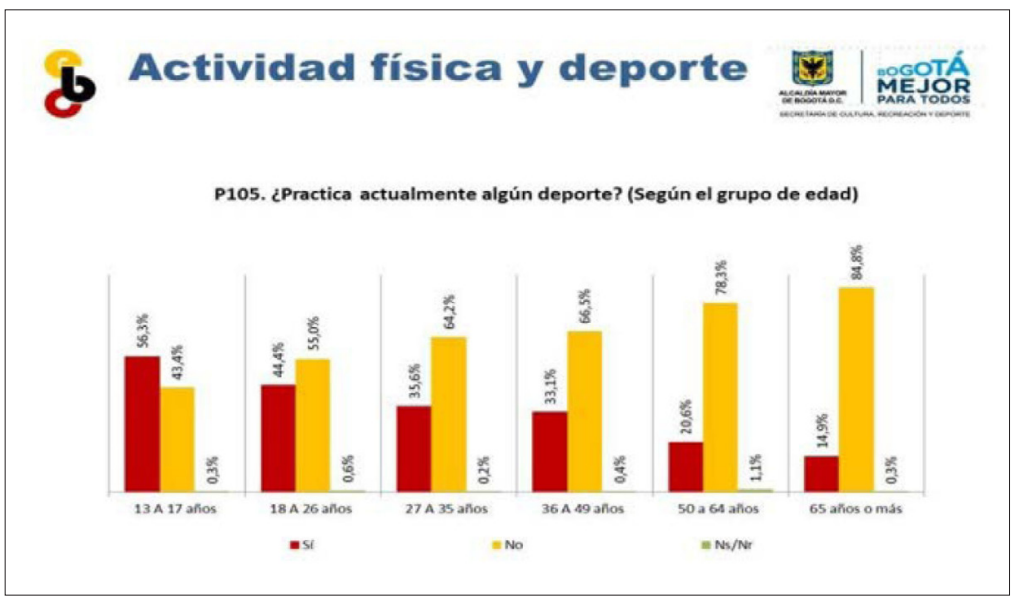

Fuente: Secretaría de Cultura, Recreación y Deporte. Encuesta Bienal de Culturas (2015). 
La estadística bienal de culturas nos dice que el 56,3\% de los adolescentes manifiesta practicar deporte, frente al 43,4\% que afirma no practicarlo. A pesar de que el porcentaje de adolescentes que realiza actividad deportiva es más alto, todavía el índice de frustración frente a la cultura del deporte en los adolescentes es alto, casi la mitad el $43,4 \%$ sigue manifestando problemáticas a nivel motor.

Por otra parte, desde el contexto de las cajas de compensación familiar, Colsubsidio muestra que sus afiliados tienen el derecho a participar con descuentos en los programas deportivos de las escuelas de formación deportiva. En este sentido, los jóvenes afiliados pueden realizar deporte si es de su interés o gusto personal en diversas disciplinas. Sin embargo, la estadística muestra lo siguiente: En Colsubsidio, para 20I4-2015 se manifiesta la afiliación de I.290.402 trabajadores, los cuales, a su vez, han afiliado a 647.548 familiares. Esto registra un total de I.937.950 personas con derecho a disfrutar de los diferentes beneficios que presta la caja de compensación familiar, incluyendo los programas deportivos. No obstante, solo I57.597 personas hacen uso de ese servicio. Estos datos estadísticamente equivalen a $8,13 \%$ de personas afiliadas que participan de los programas deportivos que presta Colsubsidio.

Figura 4. Informe de gestión y sostenibilidad Colsubsidio.

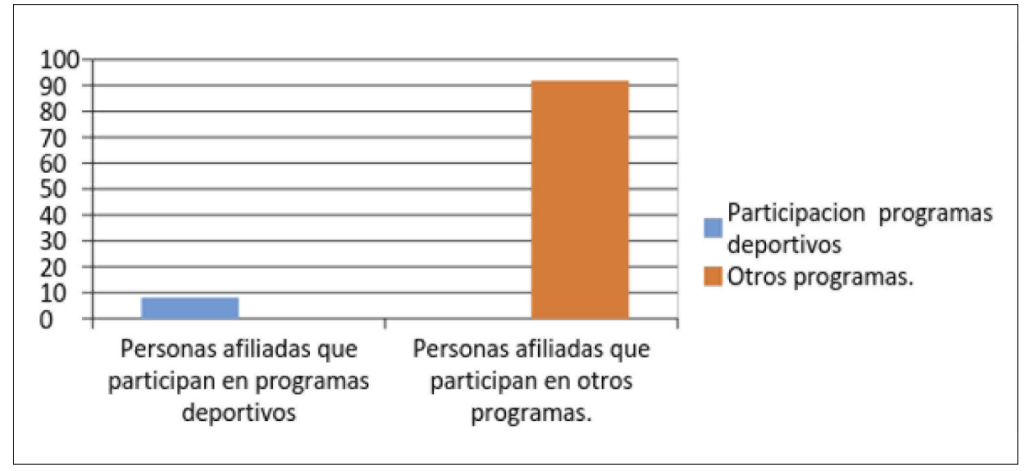

Fuente: Informe de gestión y sostenibilidad de Cafam (20I4).

En contraparte, la caja de compensación familiar de Cafam registra 861.885 trabajadores afiliados, los cuales han beneficiado a su vez a 338.165 familiares, que en total oficializa I.200.050 personas 
afiliadas que pueden hacer uso de sus servicios deportivos. De esta población afiliada, según el registro de informe de gestión y sostenibilidad de 20I5, solo 17.364 personas del grupo total afiliado participan en programas deportivos, dejando al restante de población - I.I82.686 — registrados en programas y servicios diferentes del ámbito deportivo. Estas cifras se pueden ver reflejadas en el siguiente gráfico.

Figura 5. Informe de gestión y sostenibilidad Cafam.

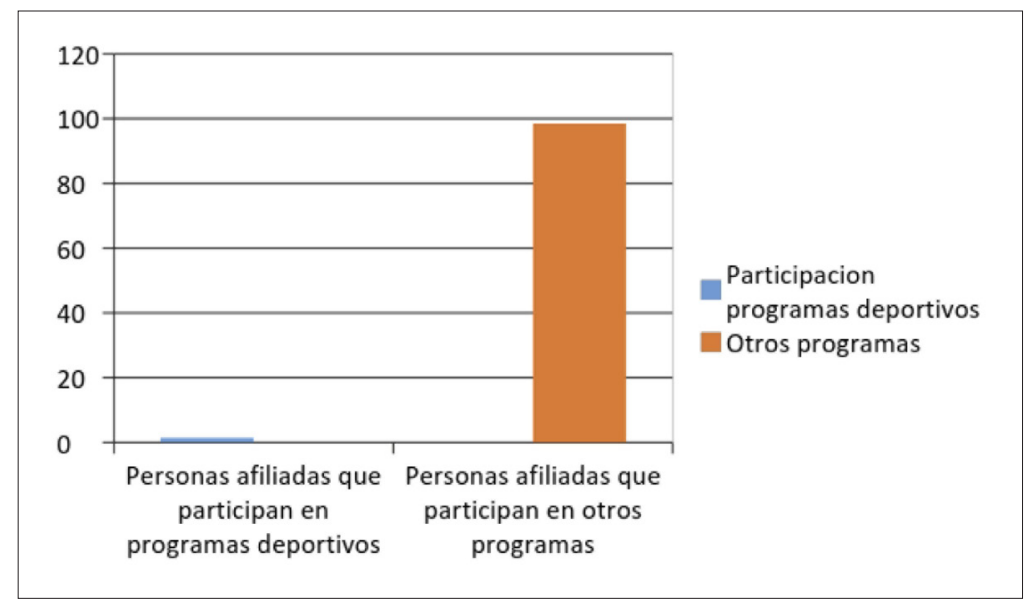

Fuente: Informe de gestión y sostenibilidad de Cafam (2015).

En síntesis, frente al problema formulado - el alejamiento de la cultura del movimiento debido a la frustración que ocasiona no poder resolver una situación motora adecuadamente-, se puede apreciar evidencia en relación con el contexto del sedentarismo vs. la práctica de actividad deportiva y los causantes; también, cifras desde la encuesta bienal de la Secretaría de Educación frente a temas de carácter deportivo y su práctica, y por último se reflejó la participación de la población en las caja de compensación familiar Colsubsidio y Cafam en programas deportivos con relación a sus trabajadores afiliados. Los anteriores escenarios reflejan la frustración motriz de las personas y su relación con la praxis del deporte.

Partiendo de esta problemática se formuló un propósito investigativo que diera solución al alejamiento de la cultura del 
movimiento y, por ende, a la falta de desarrollo motor en los adolescentes, buscando, de esta forma, mediante una estrategia didáctica, potenciar en jóvenes de media vocacional los elementos constitutivos de la motricidad, determinantes en el desarrollo de la inteligencia motriz (IM) y la resolución de situaciones motoras; una encuesta diagnóstica, que refleje los niveles de conciencia motriz frente a la eficiencia y eficacia de la inteligencia motriz; construir una guía de aprendizaje que fundamente los elementos constitutivos de la IM; aplicar un test preparatorio colectivo de los elementos constitutivos de la IM, e implementar un post test final que valide el desarrollo de la IM

Dentro de esta línea de investigación para el proyecto de inteligencia motriz, es determinante profundizar en el concepto y una de las características esenciales de la acción motriz inteligente: la motricidad entendida como una dimensión humana, en la que se consolidan sistemáticamente múltiples fenómenos, para consolidar actos de expresión y comunicación humana, en los que: "el sujeto actúa desde su realidad corpórea, y nunca se desliga de su conciencia para actuar, en otros términos los actos motores se caracterizan por arraigar en su naturaleza la intencionalidad para sentir, hacer y actuar" (Benjumea, 2009, p. 35).

De lo anterior, para la propuesta de la praxiología motriz, de Pierre Parlebas, se entiende el desarrollo de la motricidad en el ser humano como la estructuración de su inteligencia motriz, desde la premisa de que "el movimiento corporal no debe aparecer más como una mecánica al servicio de una intención, sino como un comportamiento complejo, indivisible de múltiples referencias, a la vez abstracto y concreto" (Parlebas, citado por Vizuete, 2003, p. 30). En estos términos, la inteligencia motriz es un estado consciente del movimiento humano, teniendo en cuenta sus variables y alternativas, lo cual conlleva a un comportamiento complejo que trasciende la mecanización del movimiento y la transforma en una mirada consciente de la acción motriz. "Capacidades cognitivas, de representación y decisión, aplicadas por las personas que interaccionan en situaciones sociomotrices" (Parlebas, 200I, p. 267). 
En otros términos, la inteligencia motriz, para Parlebas:

Es la conducta humana, que refiere a una "totalidad actuante" del sujeto en relación consigo mismo, con el mundo y con los otros. Una motricidad concienciada, inteligente, y por lo demás socializante, llena de significación, y por lo tanto susceptible de ser interpretada; acción que se presenta en un contexto específico el cual lo determina, hecho que a su vez permite develar una serie de informaciones provenientes del sujeto que actúa y que revelan la integridad de ese ser. (Citado por Benjumea, 2010, p. I39)

De igual manera, cabe resaltar que la conducta motriz de un practicante que, durante el desarrollo de su intervención en el espacio y el tiempo, tiene en cuenta activamente la evolución potencial de la situación, a fin de prepararse para actuar en las mejores condiciones posibles". Neurológicamente, Le Boulch propone la "acción ideomotriz" como un sistema de interiorización del movimiento, plantea un aprendizaje inteligente desde una programación consciente, "es decir, a partir de una representación mental del 'esquema de acción', lejos de resolver los problemas cotidianos con los que se enfrentan, los que enseñan el movimiento, por el contrario, los acentúa” (I982, p.II6). De esta manera, Loisel, citado por Le Boulch, descompone un acto de destreza en tres momentos claves en los procesos de la inteligencia motriz. Luego, el "Cuerpo en cambio, está sometido a una continua puesta en escena, donde en función de las exigencias del guion, asume un modelo de acción diferente cada vez que se comunica y se expresa” (Lozano, 2005, p. 76).

Por consiguiente, dicha inteligencia motriz se coloca en escena en una situación motriz; Para Parlebas (I98I, p. 7), citado por Jiménez (2012), "las situaciones motrices se clasifican según tres parámetros que son determinados por la incertidumbre, que podrá ser respecto a la información proveniente del medio físico, y/o respecto a la interacción con los compañeros y/o los adversarios". A partir de este concepto, se establece una característica esencial en las situaciones motrices, a saber, la incertidumbre, como aquel aspecto que abre mil posibilidades que pueden pasar en un evento motriz y que obligan a una buena motricidad a solucionar lo que se presente. 
Es adecuada la intervención de la praxiología motriz en la educación corporal, una propuesta desde la sociomotricidad por Parlebas (citado por Uribe, 2010). En su artículo sobre iniciación deportiva y praxiologia motriz, hace una apreciación desde el concepto escolar y propone una línea de intervención pedagógica desde el enfoque de la sociomotricidad, apuntando a la formación de un sujeto analítico, autónomo, que se adapta a las circunstancias, que soluciona problemas, que lee su contexto, que crea estrategias y es crítico-analítico-activo.

De igual manera un proyecto sobre inteligencia motriz, realizado por Paez y Forero (20I I), en relación al desarrollo de la inteligencia motriz y el juego deportivo en el contexto escolar, justifica la importancia del desarrollo de la IM y resalta el valor agregado de la comprensión de temas relevantes, como el cuerpo, la corporeidad, la motricidad, la motricidad inteligente, la descentración, la codificación, la decodificación, la sociomotricidad, la anticipación, la comunicación, las estructuras cognitivas, la acción motriz y el comportamiento motor, que dan las herramientas necesarias para afrontar problemáticas motrices.

Para los intereses de esta investigación, la IM fue concebida como un proceso complejo de interdependencia y conciencia individual y social, que el sujeto desarrolla sobre la base de los siguientes elementos constitutivos y habilidades motrices: la percepción, el acto motor, la intencionalidad, la temporalidad, la espacialidad y la corporeidad, que a su vez se dinamizan mediante características determinantes: individualidad, complejidad, simbolismo, interacción, trascendente, contextualización e historicidad, componentes que están en constante relación e interacción, como procesos cognitivos, físicos, emocionales, sociales, espaciales, que comprometen una conciencia del ser humano frente a sus acciones motrices. 
Mapa I. Elementos constitutivos y características de la motricidad

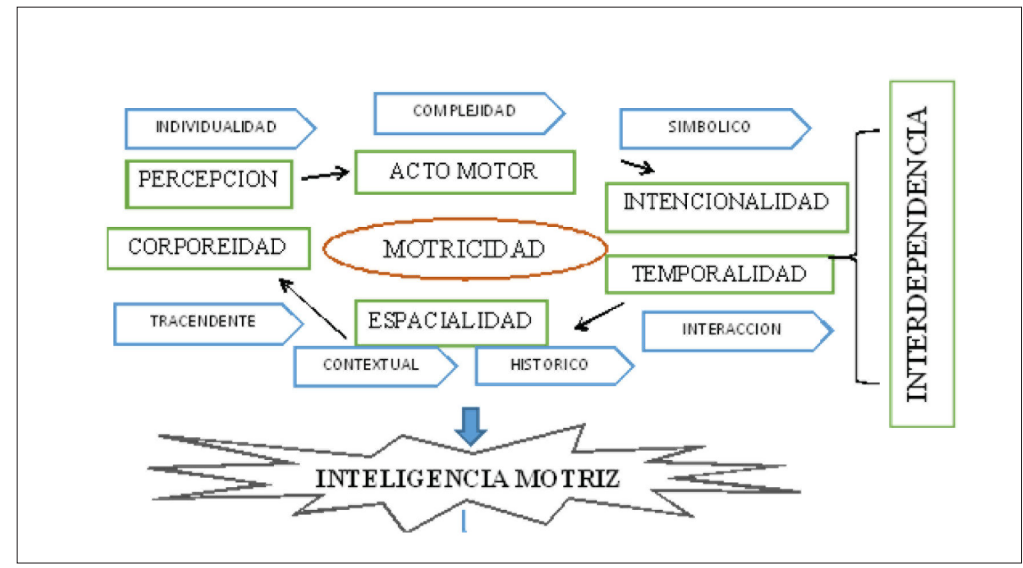

Fuente: Elementos constitutivos y características de la motricidad (Benjumea, 2009, p.178). Adaptado por Garzón (2017).

Al plantear las habilidades motrices constitutivas de la inteligencia motriz, determinantes en la resolución de situaciones motoras en jóvenes de media vocacional, es pertinente enmarcar una sustentación legal de su acción. La Constitución de I99I plantea que a toda persona se le debe garantizar la libertad de expresión (Artículo 20); además, al manifestar sus pensamientos libremente, el ciudadano también tiene derecho de asociarse en el rol de actividades sociales que él desee (Artículo 38). También promueve el ejercicio del deporte (Artículo 52), fundamental para el desarrollo de la motricidad.

Desde la Ley General de Educación se establecen pautas específicas que deben tener en cuenta los establecimientos educativos para la formación de sus adolecentes. En este horizonte, este ciclo académico debe centrarse en: la preparación de los estudiantes frente a la lectura de su cotidianidad y de los saberes ya establecidos, el fortalecimiento del lenguaje como medio de interacción y vinculación social, la preparación artística y el desarrollo de la creatividad, y el impulso de personas con actitudes investigativas (Artículo 22). De igual modo, de conformidad con la Ley de la Infancia y la Adolescencia de 2006, se debe garantizar los derechos de las niñas, niños y adolescentes. Así, su finalidad gira en torno a posibilitar ambientes armónicos de 
socialización, en los que prevalezca la igualdad y el fenómeno de la discriminación no atente contra niños y adolescentes (Artículo n ${ }^{\circ} \mathrm{I}$ ).

Dando continuidad a la coherencia y fundamentación teórica de la investigación, se implementó la estructura del proyecto mediante un modelo pedagógico acorde a sus necesidades. El modelo progresista impulsor de proyectos de vida fue el que atendió todo el proceso. Este modelo es planteado por Arboleda (2008), y busca desarrollar estructuras intelectuales de pensamiento superior en los estudiantes con las cuales ellos puedan desarrollar sus proyectos de vida por medio de una inteligencia motriz de nivel superior, que les permita solucionar problemas motores que presente la cotidianidad, no sin antes desarrollarse como personas. Para esto es necesario que se reconozcan dimensionalmente y se desarrollen proporcional y equilibradamente. Que los saberes que desarrollen sean de uso funcional en sus contextos, que sean saberes vivenciales, que los experimenten y los apropien según sus necesidades, y que los ajusten a la implementación y desarrollo de sus proyectos de vida. A lo cual Freire (1970, p. I00) agrega que "Los hombres no se hacen en el silencio, sino en la palabra, en el trabajo, en la acción, en la reflexión”.

Mapa 2. Pedagogía por proyectos de vida

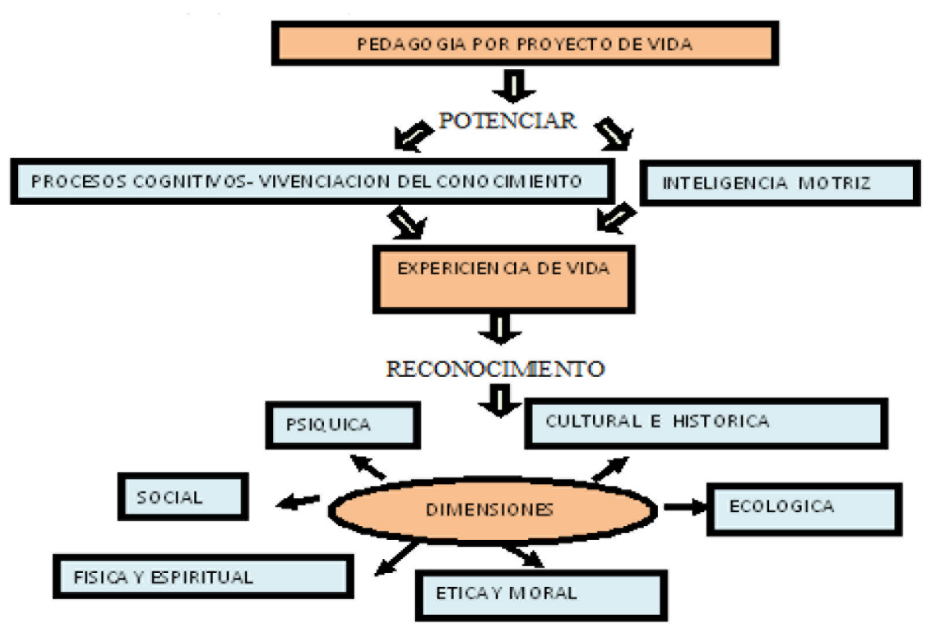

Fuente: Pedagogía por proyecto de vida. Arboleda (2007). 
Luego, la didáctica no fue menos importante dentro de la investigación. Se asumió el ABP (Aprendizaje Basado en Problemas) como la mediación más idónea; ya que el $\mathrm{ABP}$ es "una propuesta didáctica basada en la idea de hacer que los estudiantes, para aprender, no se limiten a escuchar, sino que solucionen problemas" (Hernández, 2004, p. 90). Como propuesta educativa, el ABP asume que "la finalidad del acto educativo radica en desarrollar, en el estudiante, las habilidades necesarias para que pueda resolver problemas reales" (Hernández, 2004, p. 94).

De esta manera, el ABP se caracteriza por centrar su atención en el estudiante, y más aún, no tanto en los conocimientos que él puede adquirir, sino más bien, en los que puede construir, buscando que lo aprendido pueda ser puesto en práctica en la vida cotidiana. En otras palabras, el ABP se caracteriza "por privilegiar las competencias operacionales como contenidos de aprendizaje, el ABP es una didáctica contemporánea funcional” (Hernández, 2004, p. 9I). Por otro parte, Howard (I980, p. 8) añade que el ABP tiene dos grandes virtudes — que, de manera certera, facilitaron la consecución de los propósitos educativos del proyecto investigativo-: mejora la creatividad de los estudiantes y desarrolla la habilidad para solucionar problemas.

\section{Método}

La investigación implementada para este proyecto fue de carácter evaluativo. El tipo de investigación evaluativa según (McMillan y shumacher, 2005, p. 3I), "se ocupa de una práctica concreta en una situación o situaciones determinadas. El enfoque investigativo ejecutado fue mixto; dialógico entre lo cualitativo y lo cuantitativo; es la articulación de las características, procesos y funciones de cada uno; busca generar un comunicación precisa entre las técnicas y métodos a la hora de plantear problemas y generar formas de solución. Ahora bien, a pesar de que el carácter del proyecto investigativo se basa en el enfoque cualitativo en cuanto al análisis de la información, se hizo uso de instrumentos de intervención y recolección de la información desde el enfoque cuantitativo, mediante la técnica de recolección de información o diseño investigativo cuasi-experimental (Hernández, 
2014), el cual se aplicó en el proyecto de manera correlacionada entre el rendimiento de los sujetos en su inteligencia motriz desde un test preparatorio (pre-test) y su evolución posterior en un test final (post-test) desde sus desarrollo en sus niveles de inteligencia motriz. Sin embargo, la lectura de la información fue netamente cualitativa desde los resultados estadísticos obtenidos.

Los instrumentos usados en el proyecto fueron: un test diagnóstico que dio cuenta del conocimiento general de la población con relación al objeto de estudio del proyecto; una guía de aprendizaje mediada por un seguimiento de una rúbrica de evaluación, un test de reconocimiento de la temática central y un post test de validación para efectos de verificación del producto final, en el que la interpretación buscó en primera instancia identificar una transformación en la población que les diera herramientas para su desenvolvimiento social con relación al propósito del proyecto: la potenciación de la inteligencia motriz para la resolución de problemas.

En congruencia, el método seleccionado para la investigación fue la investigación-acción (IA). Según Hernández (20I4), es un diseño investigativo que pretende unas fases cíclicas o en espiral, con las cuales el investigador puede actuar, pensar y observar. Las perspectivas en este método son técnico-científicas, deliberativas y emancipadoras; presenta posibilidades de aplicar técnicas científicas que a su vez son flexibles, pero su finalidad es emancipar o dar herramientas liberadoras a la población intervenida.

La población determinada para la investigación estuvo compuesta por adolescentes de media vocacional; una población de estudiantes de bachillerato de grado IIA, sus edades oscilaron entre I5 a 17 años, II mujeres y 17 hombres, para un total de 28 estudiantes. Los estudiantes son del colegio CELCO San Lucas, que basa su filosofía en el pensamiento del reformador Martin Lutero. El colegio presenta como proyecto educativo la formación para la vida y encamina su misión desde un enfoque educativo basado en los valores cristianos y una preparación integral. Por otra parte, la población seleccionada se enmarca en el quinto ciclo, según el reordenamiento de ciclos de la Secretaria de Educación Nacional (2008-2012). Agrupa jóvenes de I5 a $I 7$ años, que corresponden a jóvenes del grado I I. En este ciclo 
se fortalece la capacidad investigativa, de definición, interpretación, análisis, sistematización y proposición de soluciones a problemas cotidianos.

Desde este marco poblacional, en la actualidad la adolescencia centra su interés en un mundo globalizado; permanece conectada con la sociedad de consumo, debido a este contexto mediado por el internet. Vive una temporalidad agitada y acelerada, se centra en la inmediatez cotidiana; asimismo, asiste a una juvenilización social, construye y crea sus propias subculturas juveniles: formas de hablar, de vestir, de actuar, tipos de música, deportes y demás actividades que lo representan y lo constituyen. La juventud lo cuestiona todo, tiene un espíritu de riesgo, de valentía, y una capacidad creadora que responde a las necesidades del medio, se adapta fácilmente y tiene una flexibilidad que le permite imponerse al mundo. En fin, posee innumerable oportunidades y alternativas que le ofrece el mundo globalizado, y supera las generaciones anteriores debido a la gran posibilidad que tiene de conocer el mundo (Páez, 2012).

Desde lo cognitivo, para Piaget, (1966) el joven se encuentra en una etapa de operaciones formales, en la que adquiere la capacidad para pensar de manera abstracta. Desde lo moral, para Kohlberg, citado por Palomino (1989), el sujeto se encuentra en una etapa de derechos previos y contrato social, en la que actúa como una persona racional frente a los valores y realiza acuerdos y compromisos. Desde lo psicosocial, para Ekicson, citado por Bordignon (2005), el adolescente se encuentra en un estadio de dualidad entre su intimidad vs. confusión, y su intimidad vs. aislamiento; es una etapa de integración social, conformación de pares o grupos, de liderazgo y de ideología, de amistad, sexo, afiliación, cooperación y competición. Y desde el desarrollo motor, para Cabezuela y Huertas (2010), el adolescente empieza a especializar las técnicas del deporte, su tono muscular aumenta, su aparato óseo se hace más fuerte y crece, sus órganos internos crecen, adquiere más destreza física e intelectual, define su identidad sexual y desarrolla tanto su conducta motora como su personalidad.

Ahora bien, dentro del plan operativo se consolidó una unidad didáctica para el desarrollo de la inteligencia motriz, que apuntó al fortalecimiento de los 
elementos constitutivos de la inteligencia motriz como determinantes en la resolución de situaciones motoras en jóvenes de media vocacional, el cual fue implementado mediante la ejecución de una unidad didáctica, administrada por los siguientes módulos.

Tabla.I. Unidad didáctica inteligencia motriz

\begin{tabular}{|c|c|c|c|}
\hline \multicolumn{4}{|c|}{ UNIDAD DIDÁCTICA INTELIGENCIA MOTRIZ } \\
\hline $\mathrm{N}^{\mathrm{o}}$ & MÓDULOS & $\begin{array}{l}\text { SITUACIÓN } \\
\text { MOTRIZ }\end{array}$ & DESCRIPCIÓN \\
\hline $\mathrm{I}$ & Diagnóstico & $\begin{array}{l}\text { Encuesta sobre } \\
\text { inteligencia motriz }\end{array}$ & $\begin{array}{l}\text { Aplicación de un cuestionario mediante } \\
\text { preguntas cerradas, en el que se recolectará } \\
\text { información sobre el conocimiento de los } \\
\text { estudiantes con el tema del proyecto. }\end{array}$ \\
\hline 2 & Teorización & Conceptualización & $\begin{array}{l}\text { Este módulo se manejará por medio de un } \\
\text { seminario cátedra; se explicará de forma } \\
\text { teórica los conceptos de: habilidades } \\
\text { motrices, inteligencias motrices y situación } \\
\text { motriz. }\end{array}$ \\
\hline 3 & $\begin{array}{l}\text { Afianzamiento } \\
\text { motriz: cuerpo } \\
\text { protagonista }\end{array}$ & $\begin{array}{l}\text { Ejecución } \\
\text { de la guía de } \\
\text { aprendizaje para la } \\
\text { potenciación de la } \\
\text { inteligencia motriz }\end{array}$ & $\begin{array}{l}\text { Puesta en escena de la guía de aprendizaje } \\
\text { de inteligencia motriz, con el fin de } \\
\text { desarrollar las habilidades motrices } \\
\text { fundamentales para la resolución de } \\
\text { problemas a nivel motor. }\end{array}$ \\
\hline 4 & $\begin{array}{l}\text { Retroalimentación } \\
\text { grupal. } \\
\text { Preevaluación. }\end{array}$ & $\begin{array}{l}\text { Juegos } \\
\text { problémicos, } \\
\text { rubrica de } \\
\text { evaluación y test } \\
\text { preparatorio de } \\
\text { inteligencia motriz }\end{array}$ & $\begin{array}{l}\text { Desarrollo de estructuras de juegos } \\
\text { propuestos en la guía de aprendizaje, } \\
\text { construcción de un esquema lúdico en } \\
\text { el que se aplique la inteligencia motriz } \\
\text { y sus componentes, test preparatorio de } \\
\text { retroalimentación. }\end{array}$ \\
\hline 5 & $\begin{array}{l}\text { Manifestación de la } \\
\text { inteligencia motriz }\end{array}$ & $\begin{array}{l}\text { Situación motriz } \\
\text { problematizada } \\
\text { Agilidad de } \\
\text { desplazamiento } \\
\text { (códigos) } \\
\text { Presión e impacto. } \\
\text { Test de inteligencia } \\
\text { motriz }\end{array}$ & $\begin{array}{l}\text { Se presentará a la población una situación } \\
\text { problematizada, en la que deba usar las } \\
\text { habilidades motrices que componen la IM } \\
\text { para ejecutar el circuito motor y solucionar } \\
\text { las problemáticas presentadas. }\end{array}$ \\
\hline
\end{tabular}

Fuente: Adaptada por William Garzón (2019).

\section{Resultados}

A continuación, se presentan los resultados del post test o test final de la investigación realizada sobre el proyecto de inteligencia 
motriz. Para esta prueba se evaluaron los elementos constitutivos de la inteligencia motriz: percepción, intencionalidad, espacialidad, temporalidad, corporeidad y acto motor. Estas habilidades se validaron por medio de tres situaciones o problemáticas motrices; cada una evaluó dos elementos constitutivos. Por otra parte, se implementó un análisis por niveles, lo cual clasificó la población por niveles: inferior, bajo, básico, alto e inferior. El porcentaje general de los elementos constitutivos de la inteligencia motriz, en relación con sus niveles de desarrollo obtenidos, fueron:

Figura 6. Resultado estadístico porcentaje en niveles del post test de IM

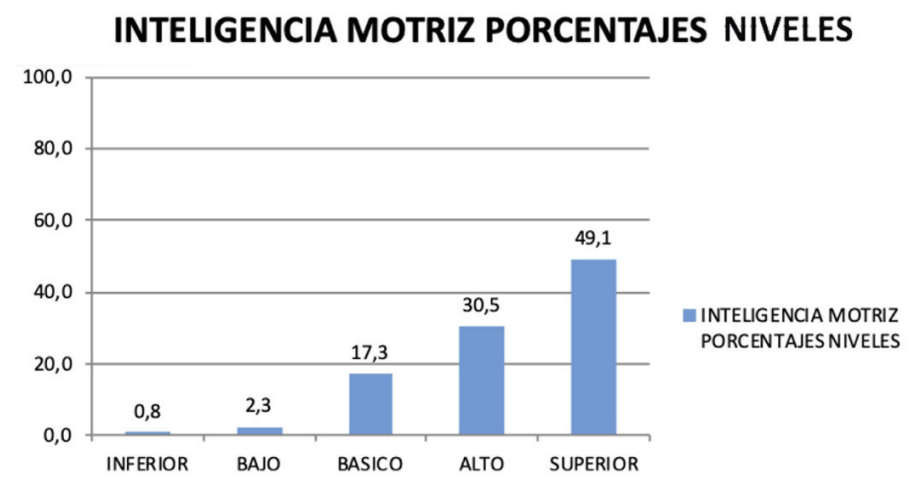

Fuente: Resultados generales de niveles de la aplicación del post test de inteligencia motriz del grado IIA.

Con relación a los anteriores resultados obtenidos, se realizó una tabulación de la información adquirida de los porcentajes de la inteligencia motriz con relación a sus niveles de desarrollo. Dicho análisis se trianguló desde el concepto de la inteligencia motriz desde lo epistemológico, el nivel de desarrollo, el resultado y el impacto en la población intervenida. 


\section{Tabla. 2 Tabulación de inteligencia motriz porcentajes de niveles.}

\begin{tabular}{|c|c|c|}
\hline \multicolumn{3}{|c|}{ CONCEPTO DE INTELIGENCIA MOTRIZ } \\
\hline \multicolumn{3}{|c|}{$\begin{array}{l}\text { Desde la perspectiva de las dimensiones intelectual, creativa y cognitiva que menciona Munera (2012), } \\
\text { el desarrollo de la inteligencia motriz, generará un pensamiento crítico, reflexivo e intencional de las } \\
\text { acciones motrices, se fortalecerá la capacidad creativa del deportista y se ampliarán sus estructuras } \\
\text { neuronales para la construcción de estrategias deportivas y cotidianas necesarias en la soluciones de } \\
\text { problemas motrices (Parlebas, citado por Vizuete, 2003, p. 30). En estos términos, la inteligencia } \\
\text { motriz es un estado consciente del movimiento humano, teniendo en cuenta sus variables y alternativas, } \\
\text { lo cual conlleva a un comportamiento complejo que trasciende la mecanización del movimiento y la } \\
\text { transforma en una mirada consciente de la acción motriz. }\end{array}$} \\
\hline NIVEL & RESULTADO & IMPACTO \\
\hline INFERIOR & $\begin{array}{l}\text { El porcentaje } \\
\text { obtenido con } \\
\text { relación al nivel } \\
\text { inferior en el } \\
\text { desarrollo del } \\
\text { acto motor fue } \\
\text { del 0,8\%. }\end{array}$ & $\begin{array}{l}\text { El porcentaje en niveles de la inteligencia motriz articula } \\
\text { el desarrollo de todos los elementos que la constituyen. } \\
\text { Para el nivel inferior el dato estadístico de la población } \\
\text { categorizada en este nivel fue realmente bajo, tan solo el } \\
0,8 \% \text { de los estudiantes determinaron, con su resultado del } \\
\text { test final, estar en un nivel de desarrollo inferior. Por un } \\
\text { lado, es un desafio para los intereses del proyecto ajustar } \\
\text { un mayor impacto en la potenciación de la inteligencia } \\
\text { motriz, para lograr en una próxima implementación que } \\
\text { ningún estudiante quede posicionado en este nivel. Por otra } \\
\text { parte, presenta una gran satisfacción frente al propósito del } \\
\text { proyecto, debido a que es muy bajo el porcentaje, lo cual } \\
\text { afirma la buena estructura de la metodología usada y un } \\
\text { balance bastante positivo. }\end{array}$ \\
\hline BAJO & $\begin{array}{l}\text { El porcentaje } \\
\text { obtenido con } \\
\text { relación al } \\
\text { nivel bajo en el } \\
\text { desarrollo del } \\
\text { acto motor fue } \\
\text { del 2,3\%. }\end{array}$ & $\begin{array}{l}\text { El porcentaje en niveles de la inteligencia motriz configura } \\
\text { el desarrollo de todos los elementos que la constituyen: } \\
\text { percepción, espacialidad, temporalidad, intencionalidad, } \\
\text { corporeidad y acto motor, aspectos que garantizan la } \\
\text { capacidad para solucionar problemas y situaciones motrices. } \\
\text { Para el nivel bajo, el resultado generado fue del } 2,3 \% \text {. Sigue } \\
\text { demostrándose la efectividad del impacto del proyecto; } \\
\text { otro porcentaje bajo de los estudiantes se ha encontrado } \\
\text { en este nivel; sin embargo, hay que fortalecer estructuras } \\
\text { del proyecto para que la población en esta categoría, en un } \\
\text { próximo impacto, escale en su nivel de desarrollo y por lo } \\
\text { menos se logre, reducir a } 0 \% \text { tanto el nivel inferior como el } \\
\text { bajo. }\end{array}$ \\
\hline BÁSICO & $\begin{array}{l}\text { El porcentaje } \\
\text { obtenido con } \\
\text { relación al nivel } \\
\text { básico en el } \\
\text { desarrollo del } \\
\text { acto motor fue } \\
\text { del } 17,3 \% \text {. }\end{array}$ & $\begin{array}{l}\text { El porcentaje en básico obtenido de la inteligencia motriz } \\
\text { se refiere al desarrollo de la intencionalidad, espacialidad, } \\
\text { percepción, corporeidad temporalidad, y acto motor. Para } \\
\text { el nivel básico, el porcentaje conseguido fue del } 17,3 \% \text {. } \\
\text { Este grupo poblacional se puede dar por satisfecho frente } \\
\text { al desarrollo de su IM; el nivel básico ya da estructuras } \\
\text { sólidas en los elementos constitutivos a la hora de enfrentar } \\
\text { problemas motrices. }\end{array}$ \\
\hline
\end{tabular}




\begin{tabular}{|c|c|c|}
\hline ALTO & $\begin{array}{l}\text { El porcentaje } \\
\text { obtenido con } \\
\text { relación al } \\
\text { nivel alto en el } \\
\text { desarrollo del } \\
\text { acto motor fue } \\
\text { del } 30,5 \% \text {. }\end{array}$ & $\begin{array}{l}\text { Para solucionar problemas y situaciones motrices. El } \\
\text { porcentaje en niveles de la inteligencia motriz desarrolla } \\
\text { todos los elementos que la determinan: corporeidad, } \\
\text { espacialidad, temporalidad, intencionalidad, percepción } \\
\text { y acto motor. Para el nivel alto se empieza a dilucidar el } \\
\text { impacto positivo y el verdadero éxito del proyecto, el 30,5\% } \\
\text { de la población consiguió establecerse en este nivel, el cual } \\
\text { ya afina los procesos motrices y aumenta el nivel de eficacia } \\
\text { y efectividad dentro de la interacción con las situaciones } \\
\text { motrices. }\end{array}$ \\
\hline SUPERIOR & $\begin{array}{l}\text { El porcentaje } \\
\text { obtenido con } \\
\text { relación al nivel } \\
\text { superior en el } \\
\text { desarrollo del } \\
\text { acto motor fue } \\
\text { del } 49,1 \% \text {. }\end{array}$ & $\begin{array}{l}\text { El porcentaje en niveles de la inteligencia motriz consolida } \\
\text { el desarrollo de todos los elementos que la constituyen: } \\
\text { acto motor, espacialidad, temporalidad, corporeidad, } \\
\text { intencionalidad y percepción, aspectos que garantizan la } \\
\text { capacidad para solucionar problemas y situaciones motrices. } \\
\text { Para el nivel superior, realmente se convierte en la satisfacción } \\
\text { total del éxito del proyecto. Presentando un porcentaje } \\
\text { del } 49 \text {,I\%, casi el } 50 \% \text { de la población logró llegar a este } \\
\text { nivel, lo que habla muy bien del proyecto, teniendo en } \\
\text { cuenta que el } 30 \% \text { fue nivel alto. Luego, casi el } 80 \% \text { de la } \\
\text { población, un porcentaje bastante alto, logró acomodarse en } \\
\text { los mejores niveles del desarrollo de la inteligencia motriz, } \\
\text { argumento que ratifica el impacto positivo del proyecto en } \\
\text { los estudiantes y al alcance de los objetivos propuestos al } \\
\text { inicio de la propuesta del desarrollo de la inteligencia motriz. } \\
\text { Se vuelve un reto para el proyecto en el próximo impacto } \\
\text { lograr que el } 30 \% \text { que esta en alto y el I7\% que está en } \\
\text { básico logren, por qué no, rankearse en el nivel superior. } \\
\text { Esto se conseguirá con un plan de mejoramiento general en } \\
\text { las debilidades que presentó el proyecto y el mejoramiento } \\
\text { de actividades desarrolladas que, mejor direccionadas y con } \\
\text { más tiempo de desarrollo, pueden brindar un mayor alcance. } \\
\text { Cabe agregar que la implementación del proyecto fue tan } \\
\text { solo de un bimestre de desarrollo: poco tiempo para alcanzar } \\
\text { mejores resultados. }\end{array}$ \\
\hline
\end{tabular}

Fuente: elaboración propia (2019).

\section{Discusión y conclusiones}

El proceso discursivo y de conclusiones de esta investigación se centró en tres aspectos fundamentales: los impactos del post test de IM, las percepciones de la población intervenida y sus sugerencias, y la construcción de un concepto propio de IM desde toda la experticia obtenida en la investigación, y que brinda los insumos necesarios para una creación epistemológica. Desde el contexto del post test se 
identificaron las siguientes conclusiones: el porcentaje en niveles de la inteligencia motriz articuló el desarrollo de todos los elementos que la constituyen: percepción, espacialidad, temporalidad, intencionalidad, corporeidad y acto motor, aspectos que garantizan la capacidad para solucionar problemas y situaciones motrices. Para el nivel inferior, el dato estadístico de la población categorizada en este nivel fue realmente bajo, tan solo el 0,8\%, y para el nivel bajo el resultado generado fue del 2,3\%. Estos datos demuestran, por un lado, un desafío para los intereses del proyecto, con el fin de ajustar un mayor impacto en la potenciación de la inteligencia motriz y lograr, para una próxima implementación, que ningún estudiante quede posicionado en estos niveles; por otra parte, presenta una gran satisfacción frente al propósito del proyecto, debido a que es muy bajo el porcentaje de no logro, lo cual afirma la buena estructura de la metodología usada y un balance bastante positivo.

Para el nivel básico, el porcentaje conseguido fue del 17,3\%. Este grupo poblacional se pudo dar por satisfecho, frente al desarrollo de su IM, debido a que el nivel básico genera estructuras sólidas en los elementos constitutivos a la hora de enfrentar problemas motrices. Para el nivel alto, se empezó a dilucidar el impacto positivo y el verdadero éxito del proyecto. El 30,5\% de la población consiguió establecerse en este nivel, el cual ya afina los procesos motrices y aumenta el nivel de eficacia y efectividad dentro de la interacción con las situaciones motrices. Qara el nivel superior, realmente se pudo establecer la satisfacción total del éxito del proyecto, presentando un porcentaje del 49,1\%, es decir, casi el 50\% de la población logró llegar a este nivel, lo que habla muy bien del proyecto implementado, teniendo en cuenta que el 30\% fue nivel alto.

Luego, se concluye que casi el $80 \%$ de la población alcanzo un porcentaje bastante alto, logró acomodarse en los mejores niveles del desarrollo de la inteligencia motriz y el $96,9 \%$ fue equivalente a niveles básico, alto y superior. En este sentido, este argumento estadístico ratificó el impacto positivo del proyecto en los estudiantes y el alcance de los objetivos propuestos al inicio de la propuesta del desarrollo de la inteligencia motriz. Se vuelve un reto para el proyecto, en próximas intervenciones, lograr que el 30\% que está en alto y 
el I7\% que está en básico logre ubicarse en el nivel superior. Esto se conseguirá mediante un plan de mejoramiento con relación a las debilidades que se presentaron en el proyecto, y la realización de actividades que, mejor direccionadas y con más tiempo de desarrollo, podrán brindar un mayor alcance.

Con relación a la discusión y desde el punto de vista de los estudiantes, se encontró que la ejecución motriz se ve afecta por la presión de los evaluadores y que la actitud del sujeto afecta las habilidades motrices. Se evidenció la falta de mejoramiento de algunos sujetos, a pesar de la aplicación de la guía de aprendizaje de la inteligencia motriz. Se destacó la importancia del ejemplo para la ejecución de los procesos motores, presentando como generador del éxito al docente y su manejo óptimo de la IM. Por otro lado, resaltó la capacidad autónoma, que generó la estructura y flexibilidad de la guía de aprendizaje. Se determinó, de igual manera, una relación directa entre el manejo de inteligencia emocional vs. la inteligencia motriz.

Algunos estudiantes manifestaron que faltó mayor precisión al explicar las situaciones problemáticas propuestas en el post test. De igual modo, como plan de mejoramiento, los estudiantes sugirieron agregar instrumentos de medición más precisos (evaluación), extender las sesiones de las prácticas para el desarrollo de las habilidades y asegurar la atención de los estudiantes en la importancia y el impacto de la práctica de la guía de aprendizaje en el desarrollo motriz.

Como ultima conclusión, gracias al proceso investigativo y todos sus hallazgos, vale la pena establecer un concepto propio del proyecto con relación a la inteligencia motriz:

La inteligencia motriz es la capacidad que tienen todos los seres humanos para solucionar problemas de movimiento expuestos en los diversos espacios sociales que conllevan niveles de desarrollo inferior, bajo, básico, alto y superior, con base en el desempeño y ritmo individual de cada sujeto. Dicha inteligencia, está constituida por seis elementos fundamentales: percepción, intencionalidad, espacialidad, temporalidad, corporeidad y el acto motor, los cuales actúan de manera articulada y nunca independiente. Luego, la acción motriz inteligente se determina, en una situación motora, cuando el sujeto usa sus sentidos y 
su sensibilidad para comprender el entorno de una manera apropiada y darle una interpretación (percepción); cuando el movimiento conlleva una intención y significado de lo que se está haciendo (intencionalidad); cuando se maneja el espacio de manera asertiva y se comprende tanto su funcionalidad y sus roles sociales, incluyendo de igual manera los objetos que lo componen y las variabilidades que dicho espacio le sugiere (espacialidad); cuando el manejo del tiempo es dinámico y el compás del movimiento es exacto y preciso (temporalidad); cuando existe una apropiación total del cuerpo, un conocimiento de sí mismo, del otro y del entorno (corporeidad), y, como un último momento, se evidencia cuando todas estas características expuestas se desvanecen de manera armónica en el acto motor, como aquel movimiento sentido, pensado y actuado intencionadamente durante una problemática motriz (Garzón, 2018).

\section{Referencias bibliográficas}

Arango. L. (2015). Informe de gestión y sostenibilidad de Colsubsidio. Recuperado de https://issuu.com/colsubsidio/docs/reportes_de_ gestion.

Arboleda, J. (2008). Modelos pedagógicos autónomos. Popayán: Corporación universitaria del Cauca.

Benjumea, M. (2009). Elementos constitutivos de la Motricidad como dimensión humana. Medellín-Colombia: Editorial Marín Vieco

Benjumea, M. (2009). La motricidad como dimensión humana, un enfoque transdisciplinar. España-Colombia: Colección Léeme.

Bordignon, N. (2005). El desarrollo psicosocial de Eric Erikson. El diagrama epigenético del adulto. Revista Lasallista de Investigación [en línea] 2005, 2 (julio-diciembre). Recuperado de http://www.redalyc.org/ articulo.oa?id $=69520210$

Cabezuelo, G. y Huerta, P. (2010). El desarrollo psicomotor: Desde la infancia hasta la adolescencia. Madrid: Narcea. S.A.

Congreso de Colombia. Ley I098 d 2006. El código de la infancia y la adolescencia. Título I, Capítulo I y II. Título II, Capítulo I. Artículos I, I2, 2I, 25, 30, 3 I y 42.

Constitución Política. (I99I). Capítulos I, II y V. Artículos 20, 38, 45,52, 67,71 y 95. 
Fairclough, S. \& Stratton, G. (2005). Physical education makes you fit and healthy'. Physical education's contribution to young people's physical activity levels. Health Education Research, 20(I), I4-23.

Howard. (1980). Problem-Based Learning. Medical Education. Recuperado de https://books.google.com.co.

Freire, P. (1970). Pedagogía del oprimido. Ciudad deMéxico: Siglo XXI editores.

Giraldo. L. (2015). Informe de gestión y sostenibilidad de Cafam. Recuperado de https://www.unglobalcompact.org/system/attachments / cop_2016/33328I/original/GRI-Cafam-2015.pdf?I477943408

Hernández, G. (2004). Enfoques pedagógicos y didácticas contemporáneas. Bogotá: Fondo internacional de pedagogía conceptual.

Hernández, S. (20I4). Metodología de la investigación. México: McGraw-Hill / Interamericana Editores.

Jiménez, F. (2012). Clasificaciones generales y específicas de los juegos deportivos: su funcionalidad pedagógica. Creative Commons. Recuperado de https://campusvirtual.ull.es/ocw/pluginfile. $\mathrm{php} / 3707 / \mathrm{mod}$ _resource/content/0/TEMA5Ide-I I-I2.pdf

Le Boulch, J. (1982). Hacia una ciencia del movimiento humano: Introducción a la Psicokinética. Buenos Aires: Editorial Paidós.

Lozano, D. (2005). La Ciencia de la Acción Motriz, Un paradigma en continua evolución. Revista Digital EF Deportes, Buenos Aires, IO(85). Recuperado de http://www.efdeportes.com

McMillan, J. y Schumacher S. (2005). Investigación educativa. Madrid: Pearson educación, S. A.

Ministerio de Educación Nacional. Ley II5 de 1984. Ley general de educación. Bogotá: Arfo. Artículos I, 5,22 y 23.

Páez. C. (2012). Los jóvenes actuales. Recuperado de http:// losinteresesdelosjovenes.blogspot.com.co.

Paez, N. \& Forero, J. (20II). Potenciación de la inteligencia motriz a través del juego deportivo. Revista digital: Corporeizando, l(6).

Palomo, A. (I989). Teoría y práctica del desarrollo moral en la escuela. Recuperado de Dialnet-LaurenceKohlberg-I 176I5.pdf.

Parlebas, P. (200I). Juegos, deporte y sociedad: Léxico de praxiología motriz. Barcelona: Editorial Paidotribo.

Piaget, J. (I966). Análisis de la Psicología genética. Buenos Aires. Recuperado de http://www.clasesatodahora.com.ar 
S.E.N. (2012-2018). Ambientes de aprendizaje reorganización curricular por ciclos. Secretaria de Educación Nacional. Recuperado de http://repositoriosed.educacionbogota.edu.co/jspui/ bitstream/I23456789/208I/I/aareorganizacionporciclosI.pdf

Secretaría de Cultura, Recreación y Deporte. Encuesta bienal de culturas (2015). Recuperado de http://www.culturarecreacionydeporte.gov.co/es/ observatorio-de-culturas/encuesta-2015

Sotomayor, P., Pineda, V., Jáuregui, O. \& Castillo, M. (20I4). Actividad física y sedentarismo: Determinantes sociodemográficos, familiares y su impacto en la salud del adolescente. Rev. Salud Pública, 16(2): I6I-I72. Recuperado de http://www.scielosp.org/pdf/rsap/vI6n2/vI6n2a0I.pdf

Uribe, I. (2010). Iniciación deportiva y Praxiología motriz. Revista de Educación Física, $29(2), 45-89$.

Valter R. et al. (2016). Motor Coordination Correlates with Academic Achievement and Cognitive Function in Children. Frontiers in Psychology, 7, 318. Recuperado de https://www.ncbi.nlm.nih.gov/ pmc/articles/PMC4792I86/ 